\title{
Separating equilibrium in quasi-linear signaling games
}

Citation for published version (APA):

Lee, J., Müller, R. J., \& Vermeulen, A. J. (2014). Separating equilibrium in quasi-linear signaling games. Maastricht University, Graduate School of Business and Economics. GSBE Research Memoranda No. 026 https://doi.org/10.26481/umagsb.2014026

Document status and date:

Published: 01/01/2014

DOI:

10.26481/umagsb.2014026

Document Version:

Publisher's PDF, also known as Version of record

\section{Please check the document version of this publication:}

- A submitted manuscript is the version of the article upon submission and before peer-review. There can be important differences between the submitted version and the official published version of record.

People interested in the research are advised to contact the author for the final version of the publication, or visit the DOI to the publisher's website.

- The final author version and the galley proof are versions of the publication after peer review.

- The final published version features the final layout of the paper including the volume, issue and page numbers.

Link to publication

\footnotetext{
General rights rights.

- You may freely distribute the URL identifying the publication in the public portal. please follow below link for the End User Agreement:

www.umlib.nl/taverne-license

Take down policy

If you believe that this document breaches copyright please contact us at:

repository@maastrichtuniversity.nl

providing details and we will investigate your claim.
}

Copyright and moral rights for the publications made accessible in the public portal are retained by the authors and/or other copyright owners and it is a condition of accessing publications that users recognise and abide by the legal requirements associated with these

- Users may download and print one copy of any publication from the public portal for the purpose of private study or research.

- You may not further distribute the material or use it for any profit-making activity or commercial gain

If the publication is distributed under the terms of Article $25 \mathrm{fa}$ of the Dutch Copyright Act, indicated by the "Taverne" license above, 


\section{Maastricht University}

Jiwoong Lee, Rudolf Müller, Dries Vermeulen

Separating equilibrium in quasi-linear signaling games

$\mathrm{RM} / 14 / 026$

\section{GSBE}

Maastricht University School of Business and Economics

Graduate School of Business and Economics

P.O Box 616

NL-6200 MD Maastricht

The Netherlands 


\title{
Separating equilibrium in quasi-linear signaling games
}

\author{
JiWoong LeE * RUdolf MÜller ${ }^{\dagger}$ \\ DRIES VERMEULEN $\ddagger$
}

June 11, 2014

\begin{abstract}
Using a network approach we provide a characterization of a separating equilibrium for standard signaling games where the sender's payoff function is quasi-linear. Given a strategy of the sender, we construct a network where the node set and the length between two nodes are the set of the sender's type and the difference of signaling costs, respectively. Construction of a separating equilibrium is then equivalent to constructing the length between two nodes in the network under the condition that the response of the receiver is a node potential.

We show that, when the set of the sender's type is finite, the collection of separating signaling functions forms a lower bounded lattice. We describe an algorithm to compute separating equilibrium strategies. When the set of the sender's type is a real interval, shortest path lengths are antisymmetric and a node potential is unique up to a constant. A strategy of the sender in a separating equilibrium is characterized by some differential equation with a unique solution.

Our results can be readily applied to a broad range of economic situations, such as the standard job market signaling model of Spence (a model not captured by earlier papers) and principal-agent models with production.
\end{abstract}

JEL Codes. C72, D82.

Keywords. Signaling game, separating equilibrium, node potential

\footnotetext{
*j.lee@keei.re.kr. Korea Energy Economics Institute, Uiwang-si, South Korea.

r.muller@maastrichtuniversity.nl Maastricht University, School of Business and Economics, Dept. of Quantitative Economics, Maastricht, The Netherlands.

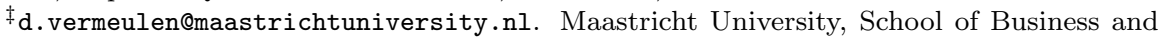
Economics, Dept. of Quantitative Economics, Maastricht, The Netherlands., P.O. Box 616, 6200 MD Maastricht, The Netherlands.
} 


\section{Introduction}

A signaling game refers to a two-player game of incomplete information in which one player has private information - a type - and the other is affected by the information. The informed player, the sender, can send a signal, contingent on type, and the uninformed party, the receiver, takes an action, which may be conditional on the observed signal. In equilibrium, the informed player optimally chooses a signal, knowing that the uninformed player will take an optimal action conditional on his inferences from the signal and the inferences will turn out to be correct. If the inferences of the uninformed party leaves no uncertainty about the informed player's type, then the equilibrium is called separating. In a separating equilibrium, the sender will deliver a type by type signal. Among many equilibria, separating equilibria are of central interest because informational asymmetry is resolved.

Signaling games arise in many economic settings. For example, in the context of industrial organization, firms may use their effort spent on advertising and launching new products to signal the quality of their product. In auction theory, an auctioneer with private information about the object's quality may put a reserve price as signal, taking a risk that an item in sale may not be sold in the end (Cai et al. [1]). In finance, an issuer who has private information about the assets may retain a fraction of the issue to signal his private information (DeMarzo and Duffie [6]). Other examples can be found in surveys such as Riley [20] and Sobel [22]. Our own analysis is motivated and illustrated by two leading examples in the literature on economic theory. First, in the traditional example due to Spence [23], a high-productivity job seeker invests in education to distinguish himself from low-productivity ones when productivity is not directly observed by an employer. The second example is a principal-agent model with production, where the production technology of the agent is not known to the principal. The principal needs to offer a wage menu to incentivize the agent to select the optimal production level.

CONTRIBUTION In this paper, we focus on those type separating strategies of the sender that, combined with the induced optimal strategy of the receiver, form a (separating) equilibrium. Such strategies are called SE strategies. We 
characterize SE strategies in a signaling game when the type space is a subset of the real line. In case the type space is finite, we show that the collection of SE strategies forms a lattice. We formulate an algorithm to compute SE strategies, and apply our results in the principal-agent model. In case the type space is a closed halfline, we identify conditions under which a unique SE strategy exists, and describe how the unique SE strategy can be computed by solving a differential equation.

Earlier results on the characterization of separating equilibrium in signaling games, despite their seeming generality, fail to capture many simple and natural extensions of the classic job market model by Spence. We achieve full coverage of (generalizations of) Spence' model by focusing on quasi-linear settings, that is, settings in which the utility function of the sender is calculated as the payment $a$ by the receiver, subtracted the cost $c(s, t)$ of signaling $s$ when of type $t$ (in formula, $\left.U_{S}=a-c(s, t)\right)$. The advantage of quasi-linearity is that it separates the cost of signaling from the response of the receiver, thereby making the cost independent of the receivers response. This advantage translates itself into two improvements upon previous literature, which are our main results.

First, we give a complete characterization of separating equilibrium that uses only four fairly mild conditions, the most prominent one being that the cost function should satisfy decreasing differences, the algebraic version of the single crossing property. Under these conditions many of the results on separating equilibrium can already be established. For example the -intuitive - result that any separating equilibrium strategy is strictly increasing with type follows immediately. Also - when type space is finite - the lattice structure of the collection of separating equilibrium outcomes can already be established under these conditions. Remarkably, we do not need any conditions on the partial derivatives of the utility function of the sender. In fact we do not even need continuity, so that these results apply in a wide variety of settings, for example when the type space is finite.

Second, when type space is a closed halfline, we only need a further three relatively natural and straightforward conditions on the primitives of the model to guarantee existence and uniqueness of separating equilibrium. The require- 
ments on the signaling cost function are that it is continuous, and that it has a continuously differentiable and bounded partial derivative with respect to the signal. In contrast, Mailath [15] and Mailath and von Thadden [16] require the utility function of the sender to be at least twice continuously differentiable, and additionally need to impose several technical assumptions regarding signs and moduli of both first and second order derivatives. In particular, the standard job market model by Spence where the utility of the sender equals the payment by the receiver minus the reciprocal of the type falls within our requirements, while it is excluded by the conditions imposed by Mailath [15] and Mailath and von Thadden [16].

APPROACH The technique we use to establish our results is in itself worth a short discussion. We use the essentially algebraic - theory of node potentials on directed graphs to derive our results. This technique has recently been applied with much success in the, related, context of mechanism design. See for example Heydenreich et al. [9], Carbajal and Ely [2], Chung and Olszewski [4] and Kos and Messner [14] for applications of the theory of networks to mechanism design without differentiability. The algebraic nature of the theory allows us to discard conditions on the first and second order partial derivatives of the utility function of sender, the conditions that drive most of the earlier results. Our proof technique highlights that differentiability is in itself not the driving force of these results. Instead, these results are shown to rely only on the implied algebraic structure of marginal gains in type pooling versus marginal costs of signaling captured by the decreasing differences condition.

RELATED LITERATURE. Mailath [15] studies a model in which the utility function of the receiver is $C^{2}$, utility is strictly monotonic in types and actions, and strictly quasi-concave in signals, plus a technical condition on the first and second order derivatives of the utility function for the sender, called boundedness. Type space is assumed to be a compact interval, the signal space is assumed to be equal to the type space ${ }^{1}$ and the action space is the entire set of reals. Under the assumption of single crossing it is shown that an one-to-one strategy is a separating equilibrium strategy precisely when the strategy is strictly increasing and it solves a characterizing differential equation. Existence and uniqueness of

\footnotetext{
${ }^{1} \mathrm{By}$ the revelation principle this is without loss of generality.
} 
a separating equilibrium strategy is then shown under the additional assumption that the derivative of the utility function of the sender with respect to signals satisfies a boundedness condition.

Mailath and von Thadden [16] study a model in which the utility function of the sender and the best response function of the receiver under complete information are both $C^{2}$. Further, under complete information the receiver needs to have a unique best response, and the induced valuation function of the sender needs to satisfy a technical condition on both first and second order derivatives of the valuation function. Thus, the conditions on the strategy of the receiver are only implicitly formulated, in terms of the induced payoff function for the sender. They show that if a separating equilibrium strategy of the receiver is differentiable at a certain point, it is characterized by a differential equation at that point. They also show that if (1) a strategy is separating, (2) it solves the characterizing differential equation, and (3) it satisfies a version of the singlecrossing property, then that strategy is incentive-compatible. They apply their results in several examples.

Quinzii and Rochet [19] study a model where both types and signals are higher dimensional. They show for the case of linear, separable costs, that a separating equilibrium exists under assumptions of smoothness of the productivity function, plus convexity of the surplus function.

Hoppe et al. [10] study a matching market between a set of men and a set of women. Each man and each woman has private information regarding their type. The value of a match depends on both the type of the man and the woman in question. ${ }^{2}$ Signals are costly, but do not depend on type of either men or women, and are linearly deducted from utility. In this model, Hoppe et al. [10] show that there exists a single symmetric, strictly monotonic, and continuously differentiable equilibrium. They also characterize this equilibrium by means of a differential equation. However, the analysis does not rule out the existence of non-symmetric, non-monotonic, or non-differentiable equilibria, and uniqueness is not guaranteed.

\footnotetext{
${ }^{2}$ In fact it is defined as the product of both types. So, if a man is of type $y$ and a woman of type $x$, and they are matched, then this generates utility $x y$, both for him and her. Thus, total utility generated by this match is $2 x y$, equally divided over both partners.
} 
ORGANIZATION. Section 2 presents the model of signaling games and several notions of graph theory. In section 3 we discuss two applications, the job market model and a principal-agent model with production. In section 4 we fully characterize separating equilibrium in terms of node potentials for an induced length function. In section 5 we discuss the implications of our characterization in the case where type space is finite. We show that the set of SE strategies forms a closed and lower bounded sublattice of $n$-dimensional Euclidean space. We also describe an algorithm to compute SE strategies, and show how our approach can be used to solve a principal-agent problem with production. In section 6 we derive conditions to guarantee existence of a unique SE strategy in case type space is a closed halfline. We show that the unique SE strategy can be computed by solving an associated differential equation. We illustrate the power of our techniques in four applications, among those the job market model and an example of a model without differentiability. All omitted proofs are in the appendix.

\section{The model}

We consider the following standard signaling game. There are two risk neutral players, a sender and a receiver. The type space and signal space of the sender are denoted by $T$ and $S$, respectively. We assume that the type space $T$ is a subset of $\mathbb{R}_{+}$and that the signal space $S$ equals $\mathbb{R}_{+}$. Further, $T$ is assumed to have a smallest element $\underline{t}$. The action space of the receiver is $A=\mathbb{R}_{+}$.

The game proceeds as follows. Nature decides the sender's type $t \in T$, and the result is communicated to the sender only. With knowledge of his type $t$, the sender chooses a signal $s \in S$, incurring a cost $c(s, t)$ depending on both type and signal. Upon observing the sender's signal $s$, the receiver chooses an action $a \in A$. The sender receives a payoff $U_{S}(a, s, t)=a-c(s, t)$ and the receiver receives a payoff $U_{R}(a, t)$. Then the game ends.

Note that the payoff of the receiver does not depend on the signal $s$. This is called the "pure signaling case" in Quinzii and Rochet [19].

ASSUMPTIONS We adopt the following four structural assumptions.

[1] The cost function $c(s, t)$ is strictly increasing in $s$ and for each $s>0$ 
strictly decreasing in $t$. Further, for all $t, c(0, t)=0$.

[2] The cost function $c(s, t)$ satisfies decreasing differences. That is, for all $s, s^{\prime} \in S$ and $t, t^{\prime} \in T$ with $s>s^{\prime}$ and $t>t^{\prime}$ it holds that

$$
c\left(s, t^{\prime}\right)-c\left(s^{\prime}, t^{\prime}\right)>c(s, t)-c\left(s^{\prime}, t\right)
$$

[3] For every $t \in T$ there exists an $\alpha(t) \in A$ with

$$
U_{R}(\alpha(t), t)>U_{R}(a, t) \quad \text { for all } a \in A \backslash\{\alpha(t)\}
$$

In other words, for every $t \in T, \alpha(t):=\arg \max _{a \in A} U_{R}(a, t)$ is unique.

[4] The resulting function $\alpha$ is strictly increasing in $t$, and $\alpha(\underline{t})=0$.

A (pure) strategy of the sender is a mapping $\sigma: T \rightarrow S$. A (pure) strategy of the receiver is a function $\gamma: S \rightarrow A$. Since in this paper we only focus on pure strategies, we omit the prefix "pure" from now on.

Definition A strategy pair $(\sigma, \gamma)$ is a separating equilibrium (SE) if the strategy $\sigma$ is one-to-one and, moreover, for all $t \in T$ we have

[1] $U_{S}(\gamma(\sigma(t)), \sigma(t), t) \geq U_{S}(\gamma(s), s, t) \quad$ for all $s \in S$

[2] $U_{R}(\gamma(\sigma(t)), t) \geq U_{R}(a, t) \quad$ for all $a \in A$.

Condition [1] states that the strategy $\sigma$ is a best response for the sender given the strategy $\gamma$ of the receiver. Condition [2] states that the strategy $\gamma$ is a best response for the receiver given the strategy $\sigma$ of the sender.

If a strategy $\sigma$ of the sender is part of a separating equilibrium it is called a separating equilibrium strategy (SE strategy). The aim of this paper is to characterize the set of SE strategies ${ }^{3}$.

We end this section with a useful observation.

Proposition 2.1 Let $(\sigma, \gamma)$ be an SE. Then $(\gamma \circ \sigma)(t)=\alpha(t)$ for all $t \in T$. Further, $\sigma(\underline{t})=0$.

\footnotetext{
${ }^{3}$ Note that the analogous task for the strategies of the receiver is a triviality.
} 
Proof. Let $(\sigma, \gamma)$ be an SE. By assumption [3] and part [2] of the definition of SE, $(\gamma \circ \sigma)(t)=\alpha(t)$ for all $t \in T$.

Write $\underline{s}=\sigma(\underline{t})$. We show that $\underline{s}=0$. Suppose $\underline{s}>0$. Since $(\gamma \circ \sigma)(t)=\alpha(t)$ for all $t$, in particular $(\gamma \circ \sigma)(\underline{t})=\alpha(\underline{t})=0$ by assumption [4]. So,

$$
U_{S}(\gamma(\sigma(\underline{t})), \sigma(\underline{t}), \underline{t})=\gamma(\sigma(\underline{t}))-c(\sigma(\underline{t}), \underline{t})=0-c(\underline{s}, \underline{t}) .
$$

Since $S=\mathbb{R}_{+}$, and $\underline{s}>0$ by assumption, we can take $0 \leq s<\underline{s}$. Then, since $\gamma(s) \in A=\mathbb{R}_{+}$and $c(s, \underline{t})$ is strictly increasing in $s$,

$$
U_{S}(\gamma(s), s, \underline{t})=\gamma(s)-c(s, \underline{t})>0-c(\underline{s}, \underline{t})=U_{S}(\gamma(\sigma(\underline{t})), \sigma(\underline{t}), \underline{t}) .
$$

This contradicts the assumption that $(\sigma, \gamma)$ is an SE. Hence, $\sigma(\underline{t})=\underline{s}=0$.

\section{Motivating examples}

Many examples in the literature fall within the model specification we described in Section 2. In this section we discuss two examples in particular. The first is a natural version of the classic job market signaling model by Spence. The second is a principal-agent model with production.

\subsection{Job market signaling}

Spence [23] is the seminal paper in signaling games. In his model, a job applicant has private information about his productivity, denoted as $t$. Because the applicant's productivity is not observed by the employer, the applicant may try to signal his productivity to an employer indirectly by investing in education, denoted as $s$. Since it is less costly for high types to take the same level of education than for low types, there can be an equilibrium in which different types choose different signals.

We consider a job market model in which the ability of the worker is characterized by an attribute $t \in T=[\underline{t}, \infty)^{4}$ with $\underline{t} \geq 0$. After having observed his ability, the worker has to choose a level of education $s \in S=[0, \infty)$. The employer then observes the level $s$ of education chosen by the worker, and based on that information chooses a wage $a \in A=[0, \infty)$.

\footnotetext{
${ }^{4}$ In the original Spence model, $T=\{1,2\}$.
} 
We assume that the employer has a unique optimal wage schedule $\alpha: T \rightarrow$ $A$ that is increasing in the observed level of education, and $\alpha(\underline{t})=0$. The assumption of increasing wages is a very natural assumption in the context where a higher level of $t$ signifies higher ability. We also assume that the cost of signaling for the worker is a separable function

$$
c(s, t)=h(s) \cdot g(t),
$$

where $h$ and $g$ are continuous functions from $S$ and $T$ respectively to $\mathbb{R}_{++}$, $h(0)=0, h$ is strictly increasing, and $g$ is strictly decreasing.

Hence, the utility to a worker is given by

$$
U_{S}(a, s, t)=a-h(s) \cdot g(t) .
$$

This model, a straightforward and natural version of the classic model by Spence, satisfies all conditions we specified in the previous section. In particular, this model automatically satisfies decreasing differences, the algebraic version of the single crossing property. Indeed, take $s, s^{\prime} \in S$ and $t, t^{\prime} \in T$ with $s>s^{\prime}$ and $t>t^{\prime}$. Then $h(s)>h\left(s^{\prime}\right)$ and $g\left(t^{\prime}\right)>g(t)$. So,

$$
\begin{aligned}
c\left(s, t^{\prime}\right)-c\left(s^{\prime}, t^{\prime}\right) & =h(s) \cdot g\left(t^{\prime}\right)-h\left(s^{\prime}\right) \cdot g\left(t^{\prime}\right) \\
& =\left(h(s)-h\left(s^{\prime}\right)\right) \cdot g\left(t^{\prime}\right) \\
& >\left(h(s)-h\left(s^{\prime}\right)\right) \cdot g(t) \\
& =c(s, t)-c\left(s^{\prime}, t\right) .
\end{aligned}
$$

Yet, this model is not covered by earlier papers such as Mailath [15] and Mailath and von Thadden [16]. For example, when $h(s)=s$, then $\frac{\partial^{2} U_{S}}{\partial s^{2}}=0$, which places this model outside the scope of those papers.

\subsection{Principal-agent model with production}

Another natural and widely applied class of signaling games is those where the type space $T$ is finite, $T=\left\{t_{1}, \ldots, t_{n}\right\}$. One application is, again, the original job market model of Spence. A second application is the principal-agent model with production. For an elaborate discussion of this application see for example Laffont and Martimort [13].

A firm, the principal, wants to delegate to an agent the production of $q$ units of a good. The value for the principal of these $q$ units is $S(q)$ where $S^{\prime}>0$, 
$S^{\prime \prime}<0$ and $S(0)=0$. The production cost of the agent is unobservable to the principal, but it is common knowledge that the fixed cost is $F$, and that the marginal cost $\theta$ belongs to the set $\Theta=\left\{\theta_{1}, \ldots \theta_{n}\right\}$, with $\theta_{1} \geq \ldots \geq \theta_{n}$. The ex ante probability that the agent has marginal $\operatorname{cost} \theta_{i}$ is $\eta_{i}$. Thus, with probability $\theta_{i}$ the agent has cost function $C\left(q, \theta_{i}\right)=F+\theta_{i} \cdot q$.

When taking his production decision the agent is informed about his marginal $\operatorname{cost} \theta$. Marginal cost $\theta$ is the agent's private information. The choice problem for the principal is to select a menu of contracts

$$
\left\{\left(p_{i}, q_{i}\right) \mid i=1, \ldots, n\right\}
$$

that separates types of the agent. So the agent with marginal $\operatorname{cost} \theta_{i}$ needs to be given the incentive to select $\left(p_{i}, q_{i}\right)$.

In this context, the type space is $T=\left\{\frac{1}{\theta_{i}} \mid i=1, \ldots, n\right\}$. By assumption in our general model, $A=S=\mathbb{R}_{+}$. Further, given the selected menu $\left\{\left(p_{i}, q_{i}\right) \mid i=\right.$ $1, \ldots, n\}$ of contracts, the response function $\alpha: T \rightarrow A$ defined by $\alpha\left(\frac{1}{\theta_{i}}\right)=p_{i}$ of the receiver (the principal) then mimics the above menu of contracts. The cost function of the sender being of type $t$ and sending signal $q$ is $c(q, t)=F+\frac{q}{t}$. So, the utility function of the sender being of type $t$, sending signal $q$, and receiving payment $p$ is

$$
U_{S}(t, q, p)=p-F-\frac{q}{t},
$$

which is indeed of the form we discussed earlier in the previous example. Therefore, this application too falls within our specification. In particular, the utility function of the sender satisfies decreasing differences.

\section{Graph representation of SE strategies}

We provide a graph theoretic interpretation of SE strategies. We recall some basic terms from graph theory. A directed graph or digraph is a pair $G=(V, E)$ where $V$ is an arbitrary set and $E$ is a collection of ordered pairs $(u, v)$ of elements $u, v \in V$ with $u \neq v$. The elements of $V$ are called nodes or vertices, and the elements of $E$ are called arcs. The complete digraph is the digraph $G=(V, E)$ where $E$ is the set of all possible ordered pairs of distinct elements of $V$. 


\subsection{The characterization}

Let $G=(V, E)$ be a complete digraph. An arc length on $G$ is a function $l: E \rightarrow \mathbb{R}$. A function $p: V \rightarrow \mathbb{R}$ is called a node potential for arc length $l$ on $G=(V, E)$ if for all $\operatorname{arcs}(u, v) \in E$

$$
p(v)-p(u) \leq l(u, v) .
$$

Let $\sigma$ be a strategy of the sender. Let $H=(T, Q)$ be the complete digraph on the type space $T$, so $Q=\{(u, v) \in T \times T \mid u \neq v\}$. Define the arc length $l(\sigma)$ on $H$ by, for all $t, t^{\prime} \in T$,

$$
l(\sigma)\left(t, t^{\prime}\right)=c\left(\sigma\left(t^{\prime}\right), t\right)-c(\sigma(t), t)
$$

Theorem 4.1 A strategy $\sigma$ of the sender is an SE strategy if and only if $\sigma$ is one-to-one, $\sigma(\underline{t})=0$, and $\alpha$ is a node potential for $l(\sigma)$ on $H$.

Proof. We show both implications separately.

A. Let $(\sigma, \gamma)$ be an SE. Since $\sigma$ is separating, it is one-to-one. Further, by Proposition 2.1, $\sigma(\underline{t})=0$. Also by Proposition 2.1, $(\gamma \circ \sigma)(t)=\alpha(t)$ for all $t$.

Using [1] of the definition of separating equilibrium, for all $t$ and $s$

$$
\alpha(t)-c(\sigma(t), t)=(\gamma \circ \sigma)(t)-c(\sigma(t), t) \geq \gamma(s)-c(s, t) .
$$

Substitution of $s=\sigma\left(t^{\prime}\right)$ and rewriting then yields

$$
\alpha\left(t^{\prime}\right)-\alpha(t) \leq c\left(\sigma\left(t^{\prime}\right), t\right)-c(\sigma(t), t)=l(\sigma)\left(t, t^{\prime}\right)
$$

for all $t, t^{\prime} \in T$. Hence, $\alpha$ is a node potential for $l(\sigma)$ on $H$.

B. Conversely, let $\sigma$ be a one-to-one strategy of the sender with $\sigma(\underline{t})=0$ and

$$
\alpha\left(t^{\prime}\right)-\alpha(t) \leq c\left(\sigma\left(t^{\prime}\right), t\right)-c(\sigma(t), t) .
$$

for all $t, t^{\prime} \in T$. Since $\sigma$ is one-to-one, for every $s \in S$ there is at most one $t \in T$ with $\sigma(t)=s$. So, we can define the strategy $\gamma$ of the receiver by

$$
\gamma(s)=\left\{\begin{aligned}
\alpha(t) & \text { if } t \in T \text { is such that } \sigma(t)=s \\
0 & \text { otherwise. }
\end{aligned}\right.
$$

It suffices to show that $(\sigma, \gamma)$ is a Nash equilibrium. First, for all $a \in A$

$$
U_{R}(\gamma(\sigma(t)), t)=U_{R}(\alpha(t), t) \geq U_{R}(a, t)
$$


by definition of $\gamma$ and $\alpha$. So, $\gamma$ is a best response of the receiver to $\sigma$.

Second, suppose the sender is of type $t$. Take $s \in S$. Suppose there exists $t^{\prime} \in T$ with $\sigma\left(t^{\prime}\right)=s$. Then $\gamma(s)=\alpha\left(t^{\prime}\right)$. So, by assumption

$$
U_{S}(\gamma(\sigma(t)), \sigma(t), t)=\alpha(t)-c(\sigma(t), t) \geq \alpha\left(t^{\prime}\right)-c\left(\sigma\left(t^{\prime}\right), t\right)=U_{S}(\gamma(s), s, t)
$$

Otherwise $\gamma(s)=0$. Then, since $\alpha(\underline{t})=0, \sigma(\underline{t})=0$, and $c(s, t)$ is increasing in $s$, from the definition of $\gamma$ and the above assumption we get

$$
\begin{aligned}
U_{S}(\gamma(\sigma(t)), \sigma(t), t) & =\alpha(t)-c(\sigma(t), t) \\
& \geq \alpha(\underline{t})-c(\sigma(\underline{t}), t) \\
& =0-c(0, t) \\
& \geq 0-c(s, t) \\
& =U_{S}(\gamma(s), s, t) .
\end{aligned}
$$

Hence, $\sigma$ is a best response for the sender to $\gamma$.

\subsection{A few useful consequences}

We need a few direct consequences of the basic characterization in the remainder of this paper. We discuss these here in this section. One direct consequence of the above characterization is the following observation.

Proposition 4.2 Let $\sigma$ be an SE strategy. Then $\sigma$ is strictly increasing in $t$.

Proof. Take $t, t^{\prime} \in T$ with $t^{\prime}>t$. To show: $\sigma\left(t^{\prime}\right)>\sigma(t)$. Since $\sigma$ is an SE strategy, by Theorem 4.1

$$
\alpha\left(t^{\prime}\right)-\alpha(t) \leq c\left(\sigma\left(t^{\prime}\right), t\right)-c(\sigma(t), t)
$$

By assumption [4] this implies $c\left(\sigma\left(t^{\prime}\right), t\right)>c(\sigma(t), t)$. Hence, since $c(s, t)$ is strictly increasing in $s$ by assumption [1], it follows that $\sigma\left(t^{\prime}\right)>\sigma(t)$.

An arc length $l$ on $H$ is strictly monotone if $l\left(t, t^{\prime}\right)+l\left(t^{\prime}, t\right)>0$ for all $t \neq t^{\prime}$. The arc length $l$ is decomposition monotone if for any $t, t^{\prime}, t^{\prime \prime} \in T$ with $t<t^{\prime}<t^{\prime \prime}$ we have $l\left(t, t^{\prime \prime}\right)>l\left(t, t^{\prime}\right)+l\left(t^{\prime}, t^{\prime \prime}\right)$ and $l\left(t^{\prime \prime}, t\right)>l\left(t^{\prime \prime}, t^{\prime}\right)+l\left(t^{\prime}, t\right)$.

Lemma 4.3 Suppose $\sigma$ is strictly increasing and $c$ has decreasing differences. Then arc length $l(\sigma)$ is strictly monotone and decomposition monotone on $H$. 
Proof. We first show that $l(\sigma)$ is strictly monotone. Take $t, t^{\prime}$ with $t<t^{\prime}$. Then $\sigma\left(t^{\prime}\right)>\sigma(t)$ by assumption. So, since $c$ has decreasing differences,

$$
l(\sigma)\left(t, t^{\prime}\right)+l(\sigma)\left(t^{\prime}, t\right)=c\left(\sigma\left(t^{\prime}\right), t\right)-c(\sigma(t), t)+c\left(\sigma(t), t^{\prime}\right)-c\left(\sigma\left(t^{\prime}\right), t^{\prime}\right)>0
$$

In order to prove that $l(\sigma)$ is decomposition monotone, take $t, t^{\prime}, t^{\prime \prime} \in T$ with $t<t^{\prime}<t^{\prime \prime}$. Then, because $\sigma\left(t^{\prime \prime}\right)>\sigma\left(t^{\prime}\right)$ and $c$ has decreasing differences,

$$
\begin{aligned}
l(\sigma)\left(t, t^{\prime \prime}\right) & =c\left(\sigma\left(t^{\prime \prime}\right), t\right)-c(\sigma(t), t) \\
& =c\left(\sigma\left(t^{\prime \prime}\right), t\right)-c\left(\sigma\left(t^{\prime}\right), t\right)+c\left(\sigma\left(t^{\prime}\right), t\right)-c(\sigma(t), t) \\
& >c\left(\sigma\left(t^{\prime \prime}\right), t^{\prime}\right)-c\left(\sigma\left(t^{\prime}\right), t^{\prime}\right)+c\left(\sigma\left(t^{\prime}\right), t\right)-c(\sigma(t), t) \\
& =l(\sigma)\left(t^{\prime}, t^{\prime \prime}\right)+l(\sigma)\left(t, t^{\prime}\right) .
\end{aligned}
$$

Similarly, we can derive that $l(\sigma)\left(t^{\prime \prime}, t\right)>l(\sigma)\left(t^{\prime}, t\right)+l(\sigma)\left(t^{\prime \prime}, t^{\prime}\right)$. Hence, $l(\sigma)$ is decomposition monotone.

\section{Characterization of SE when $T$ is finite}

Finding a separating equilibrium strategy $\sigma$ is equivalent to constructing an arc length $l(\sigma)$ so that $\alpha$ is a node potential for $l(\sigma)$ on $H$. We use this to characterize SE strategies.

\subsection{The characterization}

In this section we assume that $T=\left\{t_{1}, \ldots, t_{n}\right\}$ with $t_{i}<t_{i+1}$ for all $i=$ $1, \ldots, n-1$. We first use Theorem 4.1 to provide a further characterization for this specific setting. Next, we use the resulting characterization to construct a separating equilibrium. Define

$$
R=\left\{\left(t_{k}, t_{k+1}\right) \mid k=1, \ldots, n-1\right\} \cup\left\{\left(t_{k+1}, t_{k}\right) \mid k=1, \ldots, n-1\right\} .
$$

Theorem 5.1 A strategy $\sigma$ of the sender is an SE strategy if and only if $\sigma$ is one-to-one, $\sigma(\underline{t})=0$, and $\alpha$ is a node potential for $l(\sigma)$ on the restricted graph $(T, R)$.

Proof. The "only if" part is straightforward. To show the "if" part, assume that $\alpha\left(t^{\prime}\right)-\alpha(t) \leq l(\sigma)\left(t, t^{\prime}\right)$ for all $\left(t, t^{\prime}\right)$ in $R$. Take $\left(t_{k}, t_{k+i}\right) \in Q$ with $i \geq 2$. Since $\left(t_{k+j}, t_{k+j+1}\right) \in R$ for all $j=0, \ldots, i-1$,

$$
\alpha\left(t_{k+j+1}\right)-\alpha\left(t_{k+j}\right) \leq l(\sigma)\left(t_{k+j}, t_{k+j+1}\right) .
$$


Therefore,

$$
\begin{aligned}
\alpha\left(t_{k+i}\right)-\alpha\left(t_{k}\right) & =\sum_{j=0}^{i-1}\left(\alpha\left(t_{k+j+1}\right)-\alpha\left(t_{k+j}\right)\right) \\
& \leq \sum_{j=0}^{i-1} l(\sigma)\left(t_{k+j}, t_{k+j+1}\right) \\
& \leq l(\sigma)\left(t_{k}, t_{k+i}\right) .
\end{aligned}
$$

The last inequality follows from decomposition monotonicity of $l(\sigma)$, which in its turn follows from Proposition 4.2, assumption [2], and Lemma 4.3.

\subsection{Structure of the set of SE strategies}

In this section we show that the set of SE strategies form a lattice. We use this fact to describe an elementary algorithm to check for existence of SE strategies. Let $L$ denote the set of all non-decreasing sequences $\left(s_{1}, \ldots, s_{n}\right)$ in $S^{n}$ with $s_{1}=0$, and for all $k=1, \ldots, n-1$,

$$
c\left(s_{k+1}, t_{k+1}\right)-c\left(s_{k}, t_{k+1}\right) \leq \alpha\left(t_{k+1}\right)-\alpha\left(t_{k}\right) \leq c\left(s_{k+1}, t_{k}\right)-c\left(s_{k}, t_{k}\right) .
$$

Proposition 5.2 Let $\sigma$ be an SE strategy. Then $\left(\sigma\left(t_{1}\right), \ldots, \sigma\left(t_{n}\right)\right) \in L$. Conversely, let $s \in L$. Then $\sigma: t_{i} \mapsto s_{i}$ defines an SE strategy.

Proof. Suppose that $s=\left(s_{1}, \ldots, s_{n}\right) \in L$. Define $\sigma: T \rightarrow S$ by $\sigma\left(t_{k}\right)=s_{k}$ for all $k$. Clearly, $\sigma(\underline{t})=0$. We show that $\sigma$ is strictly increasing (and hence one-toone). Since $\alpha$ is strictly increasing by assumption [4], we have $\alpha\left(t_{k+1}\right)-\alpha\left(t_{k}\right)>$ 0 . Therefore, since $s \in L$,

$$
c\left(s_{k+1}, t_{k+1}\right)-c\left(s_{k}, t_{k+1}\right)<c\left(s_{k+1}, t_{k}\right)-c\left(s_{k}, t_{k}\right)
$$

This implies $s_{k} \neq s_{k+1}$. Then, since $s \in L$, also $s_{k+1}>s_{k}$. Further,

$$
\begin{aligned}
\alpha\left(t_{k+1}\right)-\alpha\left(t_{k}\right) & \leq c\left(s_{k+1}, t_{k}\right)-c\left(s_{k}, t_{k}\right) \\
& =c\left(\sigma\left(t_{k+1}\right), t_{k}\right)-c\left(\sigma\left(t_{k}\right), t_{k}\right) \\
& =l(\sigma)\left(t_{k}, t_{k+1}\right) .
\end{aligned}
$$

In the same way

$$
\alpha\left(t_{k}\right)-\alpha\left(t_{k+1}\right) \leq l(\sigma)\left(t_{k+1}, t_{k}\right)
$$


Hence, by Theorem 5.1, $\sigma$ is an SE strategy. The converse follows directly from Proposition 4.2 and Theorem 4.1.

Thus, in the case where $T$ is finite, characterization of the collection of SE strategies reduces to characterization of the set $L$. This is our aim in the remainder of this section.

Since $L$ is a subset of $\mathbb{R}^{n}$ it is partially ordered by the usual coordinate-wise order. For $x, y \in \mathbb{R}^{n}$, let $x \vee y \in \mathbb{R}^{n}$ be defined by $(x \vee y)_{i}=\max \left\{x_{i}, y_{i}\right\}$ and let $x \wedge y \in \mathbb{R}^{n}$ be defined by $(x \wedge y)_{i}=\min \left\{x_{i}, y_{i}\right\}$. A subset $X$ of $\mathbb{R}^{n}$ is called a sublattice of $\mathbb{R}^{n}$ when for all $x, y \in X$ also $x \vee y \in X$ and $x \wedge y \in X$.

Theorem 5.3 The set $L$ is a closed and lower bounded sublattice of $\mathbb{R}^{n}$.

Proof. Take two elements $s=\left(s_{1}, \ldots, s_{n}\right)$ and $r=\left(r_{1}, \ldots, r_{n}\right)$ in $L$. Define $x=s \wedge r$. Clearly $x$ is non-decreasing, and $x_{1}=0$. Take $k$. We show that

$$
c\left(x_{k+1}, t_{k+1}\right)-c\left(x_{k}, t_{k+1}\right) \leq \alpha\left(t_{k+1}\right)-\alpha\left(t_{k}\right) \leq c\left(x_{k+1}, t_{k}\right)-c\left(x_{k}, t_{k}\right) .
$$

If either $x_{k}=s_{k}$ and $x_{k+1}=s_{k+1}$, or if $x_{k}=r_{k}$ and $x_{k+1}=r_{k+1}$, this follows from the assumption that $s, r \in L$. Suppose that $x_{k}=s_{k}$ and $x_{k+1}=r_{k+1}$. Then we have

$$
\begin{aligned}
c\left(x_{k+1}, t_{k+1}\right)-c\left(x_{k}, t_{k+1}\right) & \leq c\left(s_{k+1}, t_{k+1}\right)-c\left(s_{k}, t_{k+1}\right) \\
& \leq \alpha\left(t_{k+1}\right)-\alpha\left(t_{k}\right),
\end{aligned}
$$

where the first inequality holds because $x_{k+1} \leq s_{k+1}, x_{k}=s_{k}$, and $c$ is strictly increasing $s$. Similarly,

$$
c\left(x_{k+1}, t_{k}\right)-c\left(x_{k}, t_{k}\right) \geq \alpha\left(t_{k+1}\right)-\alpha\left(t_{k}\right)
$$

In the same way it can be shown that $s \vee r \in L$. Hence, $L$ is a lattice. Finally, $L$ is clearly closed, and, since $L$ is subset of $\mathbb{R}_{+}^{n}$, also lower bounded.

\subsection{An algorithm}

We present a simple iterative procedure to compute an SE strategy in this setting. When $L$ is not empty, the procedure produces an SE strategy. When $L$ is empty, the procedure will detect this and decide it is not possible to compute an SE strategy. 
We start by choosing $s_{1}=0$. Now suppose that $s_{k}$ is chosen. Choose $s_{k+1} \in S$ so that

$$
\alpha\left(t_{k+1}\right)-\alpha\left(t_{k}\right) \leq c\left(s_{k+1}, t_{k}\right)-c\left(s_{k}, t_{k}\right)
$$

and

$$
\alpha\left(t_{k+1}\right)-\alpha\left(t_{k}\right) \geq c\left(s_{k+1}, t_{k+1}\right)-c\left(s_{k}, t_{k+1}\right) .
$$

It is not always possible to choose such an $s_{k+1}$. However, as soon as such a choice is possible, automatically $s_{k+1}>s_{k}$ by the assumption of decreasing differences for the cost function $c(s, t)$. Thus, the procedure generates a sequence $s=\left(s_{1}, \ldots s_{n}\right)$, and by construction $s \in L$, unless at some point the above inequalities do not admit a feasible choice for $s_{k+1}$.

Example. Consider a signaling game with $T=\{1,2,3\}$ and cost function $c(s, t)=\frac{1}{t}\left(1-\frac{1}{s+1}\right)$. First consider the case where $(\alpha(1), \alpha(2), \alpha(3))=(1,2,3)$. In this case there is no SE because the marginal cost for the sender is uniformly smaller than one, while the marginal benefit by mimicking a higher type is one, so that every type except the top will pretend to be a higher type. Our algorithm states

[1] $s_{1}=0$

[2] Choose $s_{2}>0$ such that $\frac{1}{2}\left(1-\frac{1}{s_{2}+1}\right) \leq 1 \leq\left(1-\frac{1}{s_{2}+1}\right)$.

[3] Choose $s_{3}>0$ such that $\frac{1}{3}\left(\frac{1}{s_{2}+1}-\frac{1}{s_{3}+1}\right) \leq 1 \leq \frac{1}{2}\left(\frac{1}{s_{2}+1}-\frac{1}{s_{3}+1}\right)$.

The condition in step 2 can be rewritten to $-1 \leq \frac{1}{s_{2}+1} \leq 0$, which is not feasible. Hence, the algorithm gets stuck in step 2.

Now consider the case where $(\alpha(1), \alpha(2), \alpha(3))=(0,1 / 3,1 / 2)$. The algorithm states

[1] $s_{1}=0$

[2] Choose $s_{2}>0$ such that $\frac{1}{2}\left(1-\frac{1}{s_{2}+1}\right) \leq \frac{1}{3} \leq\left(1-\frac{1}{s_{2}+1}\right)$.

[3] Choose $s_{3}>0$ such that $\frac{1}{3}\left(\frac{1}{s_{2}+1}-\frac{1}{s_{3}+1}\right) \leq \frac{1}{6} \leq \frac{1}{2}\left(\frac{1}{s_{2}+1}-\frac{1}{s_{3}+1}\right)$, or $\frac{1}{s_{2}+1}-\frac{1}{2} \leq \frac{1}{s_{3}+1} \leq \frac{1}{s_{2}+1}-\frac{1}{3}$. 
The condition in step 2 states that $\frac{1}{3} \leq \frac{1}{s_{2}+1} \leq \frac{2}{3}$. If we choose $\frac{1}{s_{2}+1}=\frac{1}{3}, s_{3}$ must be chosen such that $1 /\left(s_{3}+1\right) \in[-1 / 6,0]$ in step 3. Again, there does not exist such $s_{3}>0$ and the algorithm gets stuck.

We have an SE, though. For instance, the choice of $\frac{1}{s_{2}+1}=\frac{2}{3}$ in step 2 gives the feasible condition $1 /\left(s_{3}+1\right) \in[1 / 6,1 / 3]$ in step 3 .

Thus, in general our algorithm may get stuck, even though an SE strategy exists, and equivalently $L$ is not empty. We can avoid this irregularity by making an appropriate choice in each step of the algorithm. Since $L$ is a closed and lower bounded lattice, it has a minimum element as soon as $L$ is not empty. This minimum element of $L$ is the least costly SE strategy, and it is known as the Riley outcome. Our algorithm produces the Riley outcome when we consistently choose the lowest signal that satisfies the conditions, that is, the Riley outcome is obtained by choosing $s_{k+1}$ such that

$$
\alpha\left(t_{k+1}\right)-\alpha\left(t_{k}\right)=c\left(s_{k+1}, t_{k}\right)-c\left(s_{k}, t_{k}\right) .
$$

Finally we give a condition that guarantees existence of an SE strategy.

Assumption [E] For all $t$, the cost function $c(s, t)$ is continuous in $s$, and $c(s, t) \rightarrow \infty$ as $s \rightarrow \infty$.

Since $\alpha$ is strictly increasing, $c(s, t)$ is strictly increasing for all $t$, and $c(s, t)$ satisfies decreasing differences, by assumption [E] such a choice of $s_{k+1}$ is feasible.

\subsection{The principal-agent model with production}

We review the principal-agent model from section 3.2 and check whether the principal can select a menu

$$
\left\{\left(p_{i}, q_{i}\right) \mid i=1, \ldots, n\right\}
$$

that separates types of the agent. Thus, we need to check non-emptiness of $L$, the set of all non-decreasing sequences $\left(s_{1}, \ldots, s_{n}\right)$ in $S^{n}$ with $s_{1}=0$, and for all $k=1, \ldots, n-1$,

$$
c\left(s_{k+1}, t_{k+1}\right)-c\left(s_{k}, t_{k+1}\right) \leq \alpha\left(t_{k+1}\right)-\alpha\left(t_{k}\right) \leq c\left(s_{k+1}, t_{k}\right)-c\left(s_{k}, t_{k}\right) .
$$

This boils down to feasibility of the system

$$
\theta_{k+1} \leq \frac{p_{k+1}-p_{k}}{q_{k+1}-q_{k}} \leq \theta_{k}
$$


or alternatively

$$
\frac{p_{k+1}-p_{k}}{\theta_{k}} \leq q_{k+1}-q_{k} \leq \frac{p_{k+1}-p_{k}}{\theta_{k+1}} .
$$

From these inequalities it is clear that the principal can always choose a contract menu that separates the types of the agent, by starting with $p_{0}=q_{0}=0$, and iteratively, after having chosen $\left(p_{k}, q_{k}\right)$, choosing the next item $\left(p_{k+1}, q_{k+1}\right)$ on the menu in such a way that it satisfies the above two inequalities.

\section{Characterization of SE when $T$ is a continuum}

If the type space $T$ is a continuum, we can characterize a separating equilibrium strategy as a solution of an integral equation. The environment is the same as before except that the sender's type space is the interval $T=[\underline{t}, \infty)$. Recall that both the set of signals $S$ and the action space $A$ are $\mathbb{R}_{+}$.

ASSUMPTIONS We adopt the following three additional structural assumptions.

[5] The function $\alpha$ is continuous and $\alpha(t) \rightarrow \infty$ as $t \rightarrow \infty^{5}$.

[6] The cost function $c(s, t)$ is continuous on $S \times T$.

[7] For all $t$, the partial derivative $c_{s}(s, t)$ of $c(s, t)$ with respect to $s$ exists, and $c_{s}(s, t)$ is continuous and bounded on $S \times T$.

Proposition 6.1 The function $c_{s}(s, t)$ is non-increasing in $t$.

Proof. By assumption [2], for $h>0$ and $t \leq t^{\prime}$,

$$
c\left(s+h, t^{\prime}\right)-c\left(s, t^{\prime}\right) \leq c(s+h, t)-c(s, t) .
$$

By assumption [7] we can divide both sides by $h$ and take limits for $h \rightarrow 0$, which yields the inequality $c_{s}\left(s, t^{\prime}\right) \leq c_{s}(s, t)$.

We need the following observation in this setting.

Lemma 6.2 Suppose that $\sigma$ is an SE strategy. Then, $\sigma$ is strictly increasing and continuous.

\footnotetext{
${ }^{5}$ In fact all we need is that for all $a \in A$ there exists a $t \in T$ with $\alpha(t)=a$. Assumption [5] is simply a convenient way, but by no means the only way, to establish this.
} 
Proof. Let $(\sigma, \gamma)$ be an SE. Then $\sigma$ is strictly increasing by Proposition 4.2. We show that $\sigma$ is continuous. Take $t \in T$. Define $\sigma(t+):=\lim _{\varepsilon \downarrow 0} \sigma(t+\varepsilon)$. Since $(\sigma, \gamma)$ is an SE, for every sufficiently small $\varepsilon>0$ we have

$$
\gamma(\sigma(t+\varepsilon))-c(\sigma(t+\varepsilon), t+\varepsilon) \geq \gamma(\sigma(t))-c(\sigma(t), t+\varepsilon)
$$

Since $\gamma(\sigma(t+\varepsilon))=\alpha(t+\varepsilon)$ and $\gamma(\sigma(t))=\alpha(t)$ by Proposition 2.1, this inequality implies that

$$
c(\sigma(t+\varepsilon), t+\varepsilon)-c(\sigma(t), t+\varepsilon) \leq \alpha(t+\varepsilon)-\alpha(t) .
$$

Since $c(s, t)$ is continuous by assumption [6] and $\alpha$ is continuous by assumption [5], taking limits as $\varepsilon \downarrow 0$ yields

$$
c(\sigma(t+), t)-c(\sigma(t), t) \leq 0
$$

So, $\sigma(t+) \leq \sigma(t)$ by assumption [1], which implies that $\sigma$ is right-continuous. Similarly, $\sigma$ is left-continuous. Hence, $\sigma$ is continuous.

\subsection{The characterization}

We characterize SE strategies for the sender.

In the characterization we need the following notions. Consider a complete digraph $G=(V, E)$ on a finite set $V$ of nodes. A path is a vector $\pi=\left(v_{0}, \ldots, v_{k}\right)$ with $\left(v_{i}, v_{i+1}\right) \in E$ for all $i=0, \ldots, k-1$. For two nodes $u, v \in V$, a path from $u$ to $v$ is a path $\left(v_{0}, \ldots, v_{k}\right)$ with $v_{0}=u$ and $v_{k}=v$. For $r, t \in T$, the set of all paths from $r$ to $t$ is denoted by $\Pi(r, t)$.

Let $l$ be an arc length on $G$. The length of a path $\pi=\left(v_{0}, \ldots, v_{k}\right)$, denoted by length $(l)(\pi)$, is defined as

$$
\operatorname{length}(l)(\pi)=\sum_{i=0}^{k-1} l\left(v_{i}, v_{i+1}\right) .
$$

The distance from $r$ to $t$ with respect to the the arc length $l(\sigma)$ is defined by

$$
\operatorname{dist}(\sigma)(r, t)=\inf _{\pi \in \Pi(r, t)} \text { length }(l(\sigma))(\pi)
$$

Take $a \in A$. By assumptions [4] and [5] there exists a $t \in T$ with $\alpha(t)=a$. In other words, $\alpha^{-1}: A \rightarrow T$ exists. Take $(s, a) \in S \times A$. Define

$$
H(s, a):=c_{s}\left(s, \alpha^{-1}(a)\right)
$$


We have the following result.

Theorem 6.3 A strategy $\sigma$ is an SE strategy if and only if $\sigma=\gamma^{-1} \circ \alpha$, where $\gamma: S \rightarrow A$ is a solution to the initial value problem

$$
\gamma^{\prime}(s)=H(s, \gamma(s)) \text { with } \gamma(0)=0
$$

and $\gamma(s) \rightarrow \infty$ as $s \rightarrow \infty$

Proof. The proof is in two parts.

A. Let $\sigma$ be an SE strategy. By Lemma B.5 it follows that

$$
\alpha(t)=\int_{\underline{t}}^{t} h(\sigma)(x) d \sigma(x)
$$

where $h(\sigma)(x)=c_{s}(\sigma(x), x)$. Further, $\sigma$ is continuous and strictly increasing by Lemma 6.2. So, since $\sigma(\underline{t})=0$, either $\sigma(T)=[0, \infty)$, or there is an $s^{*}$ with $\sigma(T)=\left[0, s^{*}\right)$. Suppose the latter. We derive a contradiction. Define $\gamma(s)=\left(\alpha \circ \sigma^{-1}\right)(s)$ for all $s \in\left[0, s^{*}\right)$. Then $\gamma(0)=0$ by Proposition 2.1 and assumption [4].

Take $s \in\left[0, s^{*}\right)$. By Theorem A.1 the change of variable $y=\sigma(x)$ yields for $s=\sigma(t)$ that

$$
\gamma(s)=\left(\alpha \circ \sigma^{-1}\right)(s)=\int_{\sigma(\underline{t})}^{\sigma(t)} c_{s}\left(y, \sigma^{-1}(y)\right) d y=\int_{0}^{s} H(y, \gamma(y)) d y .
$$

Since the function $y \mapsto H(y, \gamma(y))$ is continuous by Lemma 6.2 and assumptions [5] and [7], the Fundamental Theorem of Calculus states that $\gamma$ is differentiable, and that for all $s \in\left[0, s^{*}\right)$

$$
\gamma^{\prime}(s)=H(s, \gamma(s))-H(0, \gamma(0))
$$

Since $H(0, \gamma(0))=H(0,0)=c_{s}\left(0, \alpha^{-1}(0)\right)=c_{s}(0, \underline{t})=0$, we see that $\gamma$ is a partial solution to the differential equation. By Theorem C.2 there is a unique solution $f$, and $f(s)=\gamma(s)=\left(\alpha \circ \sigma^{-1}\right)(s)$ for all $s \in\left[0, s^{*}\right)$. This however implies that for every $t \in T$

$$
\alpha(t)=\left(\alpha \circ \sigma^{-1}\right)(\sigma(t))=\gamma(\sigma(t))=f(\sigma(t)) \leq f\left(s^{*}\right) .
$$

This contradicts assumption [5]. Hence, $\sigma(T)=[0, \infty)$, and $\gamma$ coincides with the solution $f$ of the initial value problem. 
B. Conversely, let $\gamma: S \rightarrow A$ be a solution to the initial value problem

$$
\gamma^{\prime}(s)=H(s, \gamma(s)) \text { with } \gamma(0)=0 .
$$

Then $\gamma$ is differentiable and hence continuous. Further, since $\gamma^{\prime}(s)=c_{s}(s, \gamma(s))>$ 0 by assumption [1], $\gamma$ is also strictly increasing.

Further, since $\gamma(s) \rightarrow \infty$ as $s \rightarrow \infty$, for every $t$ there exists an $s$ with $\gamma(s)=\alpha(t)$.

So we can define $\sigma=f^{-1} \circ \alpha$. Then also $\sigma$ is strictly increasing and continuous by assumptions [4] and [5]. Moreover, $\sigma(\underline{t})=f^{-1}(\alpha(\underline{t}))=f^{-1}(0)=0$. So, by Theorem 4.1 it suffices to show that $\alpha$ is a node potential for $l(\sigma)$. Take $t, t^{\prime} \in T$. We show that

$$
\alpha\left(t^{\prime}\right)-\alpha(t) \leq l(\sigma)\left(t, t^{\prime}\right) .
$$

On the one hand, by definition of $\sigma$,

$$
\begin{aligned}
\alpha\left(t^{\prime}\right)-\alpha(t) & =(f \circ \sigma)\left(t^{\prime}\right)-(f \circ \sigma)(t)=\int_{\sigma(t)}^{\sigma\left(t^{\prime}\right)} f^{\prime}(x) d x \\
& =\int_{\sigma(t)}^{\sigma\left(t^{\prime}\right)} H(x, f(x)) d x=\int_{\sigma(t)}^{\sigma\left(t^{\prime}\right)} c_{s}\left(x, \alpha^{-1}(f(x)) d x\right. \\
& =\int_{\sigma(t)}^{\sigma\left(t^{\prime}\right)} c_{s}\left(x, \sigma^{-1}(x)\right) d x .
\end{aligned}
$$

On the other hand,

$$
l(\sigma)\left(t, t^{\prime}\right)=c\left(\sigma\left(t^{\prime}\right), t\right)-c(\sigma(t), t)=\int_{\sigma(t)}^{\sigma\left(t^{\prime}\right)} c_{s}(x, t) d x .
$$

Now note that, since $\sigma$ is strictly increasing, for any $x \geq \sigma(t)$ we have $\sigma^{-1}(x) \geq$ $t$. Therefore $c_{s}\left(x, \sigma^{-1}(x)\right) \leq c_{s}(x, t)$ for any $x \geq \sigma(t)$ by Proposition 6.1 . Hence,

$$
\alpha\left(t^{\prime}\right)-\alpha(t)=\int_{\sigma(t)}^{\sigma\left(t^{\prime}\right)} c_{s}\left(x, \sigma^{-1}(x)\right) d x \leq \int_{\sigma(t)}^{\sigma\left(t^{\prime}\right)} c_{s}(x, t) d x=l(\sigma)\left(t, t^{\prime}\right) .
$$

This completes the proof.

\subsection{Uniqueness of the SE strategy}

We show there is a unique SE strategy in the model where $T$ is a continuum, under the condition that the solution $\gamma^{*}$ of the differential equation has $\gamma^{*}(s) \rightarrow$ $\infty$ when $s \rightarrow \infty$. 
Theorem 6.4 The unique SE strategy is $\sigma=\left(\gamma^{*}\right)^{-1} \circ \alpha$, where $\gamma^{*}$ is the unique solution to the initial value problem

$$
\gamma^{\prime}(s)=H(s, \gamma(s)) \text { with } \gamma(0)=0 \text {. }
$$

Proof. The function $H$ is continuous, and non-increasing in the second argument due to assumptions [2], [4], and [7]. So, the initial value problem

$$
f^{\prime}(s)=G(s, f(s)) \text { with } f(0)=0
$$

has a unique solution $f^{*}$ by Theorem C.2. The above result now follows immediately from Theorem 6.3.

\section{Applications}

In this section we present a few applications and direct consequences of our results. We first discuss a simple example to illustrate the computational power of our results. The second application is the continuous-type version of the classic job market model by Spence [23] we presented in section 3.1. Thirdly, we illustrate the scope of our results in an example outside the context of differentiable utility. Finally we briefly discuss how our results contribute to the theory on mechanism design and implementation.

\subsection{Application: the differentiable case}

As a first application we consider the case where $\alpha$ is differentiable.

Corollary 7.1 Suppose $\alpha$ is differentiable on T. Then, the unique SE strategy $\sigma^{*}$ is also differentiable and its inverse $\tau=\left(\sigma^{*}\right)^{-1}$ solves the initial value problem

$$
\alpha^{\prime}(\tau(s)) \cdot \tau^{\prime}(s)=c_{s}(s, \tau(s)) \text { with } \tau(0)=\underline{t} .
$$

Proof. From Theorem 6.4 it follows immediately that the SE strategy $\sigma$ is differentiable as well. Further, by differentiating both sides in the integral expression for $\alpha$ with respect to $s$, we have the differential equation directly yielding $\sigma$.

Example. Take $\alpha(t)=t^{2}$ and $c(s, t)=\frac{s}{t+1}$. It is straightforward to check that this model satisfies all conditions [1] till [7]. Thus, the above corollary 
applies, and

$$
2 \cdot \tau(s) \cdot \tau^{\prime}(s)=\frac{1}{\tau(s)+1} .
$$

This can be rewritten to the differential equation

$$
2 \cdot \tau(s) \cdot(\tau(s)+1) \cdot \tau^{\prime}(s)=1
$$

which yields $\frac{2}{3} \tau(s)^{3}+\tau(s)^{2}=s$. It immediately follows that $\sigma(t)=\frac{2}{3} t^{3}+t^{2}$.

In fact, the same analysis applies to the situation where $\alpha$ is strictly increasing and continuously differentiable, and $c(s, t)=s \cdot g(t)$, where $g$ is any strictly positive, continuous, and strictly decreasing function on $\mathbb{R}_{+}$. Then by the above corollary

$$
\frac{\alpha^{\prime}(\tau(s))}{g(\tau(s))} \cdot \tau^{\prime}(s)=1
$$

Integrating both sides with respect to $s$ and using the substitution $z=\tau(s)$ and $d z=\tau^{\prime}(s) d s$ for the left-hand side yields

$$
\sigma(t)=(s=) \int_{0}^{t} \frac{\alpha^{\prime}(z)}{g(z)} d z .
$$

This integral immediately renders the unique (continuously differentiable) separating equilibrium strategy.

\subsection{Application: job market signaling}

Consider the following instance of the job market signaling game discussed in section 3.1. The applicant is the sender and the employer is the receiver, respectively. The type space is $T=[1, \infty)$ and the signal space is $S=\mathbb{R}_{+}$. The best action of the receiver when the sender is of type $t \in T$ is $\alpha(t)=t$, and the cost for the sender when he is of type $t \in T$ and sends signal $s \in S$ is $c(s, t)=\frac{s}{t}$.

Since this example is an instance of our model in section 3.1, we can apply our results. By Corollary 7.1, the inverse $\sigma$ of the solution $\tau$ of the differential equation

$$
\tau^{\prime}(s)=\frac{1}{\tau(s)} \quad \text { with } \tau(0)=1
$$

is the unique SE strategy. It is straightforward to check that $\tau(s)=\sqrt{2 s+1}$. Hence, $\sigma(t)=\frac{1}{2}\left(t^{2}-1\right)$ is the unique SE strategy of this job signaling model. 


\subsection{Application: the non-differentiable case}

Consider the job market model where $T=A=S=\mathbb{R}_{+}, g(t)=\frac{1}{t+1}, h(s)=s$, and

$$
\alpha(t)=\left\{\begin{array}{cl}
t & \text { if } 0 \leq t \leq 3 \\
\frac{1}{3} t+2 & \text { if } 3 \leq t
\end{array}\right.
$$

Note that $\alpha$ is continuous, but not differentiable. Nevertheless, our results apply. We first compute $H(s, a)$. Note that $c(s, t)=s \cdot g(t)$. So, $c_{s}(s, t)=g(t)$, and therefore $H(s, a)=c_{s}\left(s, \alpha^{-1}(a)\right)=g\left(\alpha^{-1}(a)\right)$. It is now easy to compute that

$$
\alpha^{-1}(a)=\left\{\begin{array}{cl}
a & \text { if } 0 \leq a \leq 3 \\
3 a-6 & \text { if } 3 \leq a
\end{array} \quad \text { and } \quad H(s, a)=\left\{\begin{array}{cl}
\frac{1}{a+1} & \text { if } 0 \leq a \leq 3 \\
\frac{1}{3 a-5} & \text { if } 3 \leq a .
\end{array}\right.\right.
$$

Thus, Theorem 6.3 states that a separating equilibrium strategy $\sigma$ is determined by $\sigma=\gamma^{-1} \circ \alpha$ where $\gamma$ solves the initial value problem that $f(0)=0$ and

$$
f^{\prime}(s)=(H(s, f(s))=) \begin{cases}\frac{1}{f(s)+1} & \text { if } 0 \leq f(s) \leq 3 \\ \frac{1}{3 f(s)-5} & \text { if } 3 \leq f(s) .\end{cases}
$$

The (unique) solution to the differential equation above is

$$
\gamma(s)= \begin{cases}\sqrt{s+1}-1 & \text { if } 0 \leq s \leq 15 \\ \frac{1}{3} \cdot(\sqrt{6 s-74}+5) & \text { if } 15 \leq s\end{cases}
$$

We get that

$$
\gamma^{-1}(a)= \begin{cases}(a+1)^{2}-1 & \text { if } 0 \leq a \leq 3 \\ \frac{1}{6} \cdot\left((3 a-5)^{2}+74\right) & \text { if } 3 \leq a\end{cases}
$$

Hence, since $\sigma=\gamma^{-1} \circ \alpha$, we find that

$$
\sigma(t)= \begin{cases}(t+1)^{2}-1 & \text { if } 0 \leq t \leq 3 \\ \frac{1}{6} \cdot\left((t+1)^{2}+74\right) & \text { if } 3 \leq t\end{cases}
$$

Finally, note that the SE strategy $\sigma$ is indeed continuous, and that, although $\gamma$ is differentiable, the SE strategy $\sigma$ is not.

\subsection{Application: incentives for truthful reporting}

Our results also have implications for mechanism design. Note that $T$ is a subset of $S$. Also note in the job market signaling application that merely the payment scheme $\alpha(t)=t$ does not induce the truthful report $\sigma(t)=t$, but $\sigma(t)=\frac{1}{2}\left(t^{2}-1\right)$ instead.

If we want $\sigma(t)=t$ for all $t \in T$, then the SE condition that $\sigma=\left(f^{*}\right)^{-1} \circ \alpha$ yields $f^{*}(t)=\alpha(t)$ for all $t \in T$. Hence, by Theorem 6.4, 
Theorem 7.2 The model allows truthful reporting in equilibrium precisely when $\alpha$ solves

$$
\alpha^{\prime}(t)=H(t, \alpha(t))=c_{s}(t, t)
$$

If we consider the signaling cost function $c(s, t)=\frac{s}{t+1}$, then $c_{s}(t, t)=\frac{1}{t+1}$, so that the differential equation $\alpha^{\prime}(t)=\frac{1}{t+1}$ yields $\alpha(t)=\log (t+1)$. Thus, in order to induce truthful reporting, the planner needs to apply the specific reward function $\alpha(t)=\log (t+1)$.

\section{Discussion}

In the context of one-dimensional signaling games, we characterized the set of separating equilibrium strategies using techniques known from network theory and mechanism design with non-differentiable utility. We showed that, in case type space is finite, the set of SE strategies forms a lattice. We constructed an algorithm to compute SE strategies, and applied our results in the context of a principal-agent model with production. In case type space is a closed halfline, we identified conditions under which there exists a unique SE strategy, and showed how to compute the SE strategy by means of a differential equation. We applied these results in several examples, including a generalization of the Spence job market model, and an example with non-differentiable primitives.

Finally we briefly compare our results to Mailath [15]. Consider the class of natural variations on the model of Spence that we discussed in section 3.1, where $\alpha$ is strictly increasing and continuously differentiable, and the cost of signaling is separable and linear in $s, c(s, t)=h(s) \cdot g(t)$. None of these models are covered by the analysis in Mailath [15] since they fail conditions (4) and (5) ${ }^{6}$ In particular, $U_{3}=g(t)$ and $U_{33}=0$, so neither strict quasiconcavity nor boundedness holds.

Another condition that fails to hold is condition (1) when $\alpha$ is only strictly increasing and continuous. Then

$$
U(t, \hat{t}, s)=\alpha(\hat{t})-h(s) \cdot g(t)
$$

So, requirement (1) of Mailath [15] that $U$ is $C^{2}$ does not necessarily hold.

\footnotetext{
${ }^{6}$ And for the same reason the more general results in Mailath and von Thadden [16] do not apply here, since (4) and (5) are equivalent to assumption 2 in that paper.
} 


\section{References}

[1] Cai H, J Riley, and L Ye (2007) Reserve price signaling. Journal of Economic Theory 135:253-268.

[2] Carbajal JC, and JC Ely (2013) Mechanism design without revenue equivalence. Journal of Economic Theory 148:104-133.

[3] Cho I.-K, and D Kreps (1987) Signaling Games and Stable Equilibria. The Quarterly Journal of Economics 102:179-222.

[4] Chung KS, and W Olsewski (2007) A non-differentiable approach to revenue equivalence. Theoretical Economics 2: 469-487.

[5] DeMarzo P (2005) The Pooling of Tranching of Securities: A Model of Informed Intermediation. Review of Financial Studies 18:307-316.

[6] DeMarzo P, and D Duffie (1999) A Liquidity-Based Model of Security Design. Econometrica 67:68-100.

[7] Hartman P (1982) Ordinary Differential equation. Birkhäuser

[8] Hellwig M (1992) Fully revealing outcomes in signalling models: An example of nonexistence when the type space is unbounded. Journal of Economic Theory 58:93-104.

[9] Heydenreich B, R Müller, M Uetz, and R Vohra (2009) Characterization of revenue equivalence. Econometrica 77:307-316.

[10] Hoppe H, B Moldovanu, and A Sela (2009) The Theory of Assortative Matching Based on Costly Signal. Review of Economic Studies 76:253-281.

[11] Jullien B, and T Mariotti (2006) Auction and the informed seller problem. Games and Economic Behavior 56:225-258.

[12] Köszegi B, and M Rabin (2006) A model of reference-dependent preferences. Quaterly Journal of Economics 121:1133-1119.

[13] Laffont JJ, and D Martimort (2001) The theory of incentives: the principalagent model. Princeton University Press. 
[14] Kos N, and Messner M (2012) Extremal incentive compatible transfers. Journal of Economic Theory 148:134-164.

[15] Mailath GJ (1987) Incentive compatibility in signaling games with a continuum of types. Econometrica 55:1349-1365.

[16] Mailath GJ and EL von Thadden (2013) Incentive compatibility and differntiability: new results and classic applications. Journal of Economic Theory (accepted).

[17] Mishra D and Talman D (2010) "Characterization of the Walrasian equilibria of the assignment model. Journal of Mathematical Economics, 46:6-20.

[18] Milgrom P (2004) Putting auction theory to work. Cambridge University Press.

[19] Quinzii M and Rochet JC (1985) Multidimensional signalling. Journal of Mathematical Economics 14:261-284.

[20] Riley JG (1979) Informational equilibrium. Econometrica 47:331-359.

[21] Rudin W (1976) Principles of mathematical analysis. International series in pure and applied mathematics. McGraw-Hill Book Co.

[22] Sobel J (Forthcoming) Signaling games. Encyclopedia of Complexity and Systems Science. Springer.

[23] Spence M (1973) Job market signaling. The Quarterly Journal of Economics $87: 355-374$.

[24] Vohra R (2011) Mechanism Design: A Linear Programming Approach. Econometric Society Monographs. Cambridge University Press

\section{A Appendix. Riemann-Stieltjes integration}

We recall the concept of the Riemann-Stieltjes integral. For $r, t \in \mathbb{R}$ with $r<t$, a partition from $r$ to $t$ is a path $\pi=\left(t_{0}, \ldots, t_{k}\right)$ with

$$
r=t_{0}<t_{1}<\cdots<t_{k-1}<t_{k}=t
$$


Let $P(r, t)$ be the collection of partitions from $r$ to $t$. Given a bounded function $f:[r, t] \rightarrow \mathbb{R}$ and a partition $\pi$ from $r$ to $t$, we define

$$
M_{i}=\sup \left\{f(t) \mid t_{i} \leq t \leq t_{i+1}\right\} \quad \text { and } \quad m_{i}=\inf \left\{f(t) \mid t_{i} \leq t \leq t_{i+1}\right\} .
$$

Let $g$ be non-decreasing on $[r, t]$. Given a partition $\pi$ from $r$ to $t$ we write $\Delta g_{i}=g\left(t_{i+1}\right)-g\left(t_{i}\right)$. Write

$$
U(\pi, f, g)=\sum_{i=0}^{k-1} M_{i} \cdot \Delta g_{i} \quad \text { and } \quad L(\pi, f, g)=\sum_{i=0}^{k-1} m_{i} \cdot \Delta g_{i}
$$

It is straightforward to check that $\inf _{\pi \in P(r, t)} U(\pi, f, g)$ and $\sup _{\pi \in P(r, t)} L(\pi, f, g)$ exist, and that

$$
\inf _{\pi \in P(r, t)} U(\pi, f, g) \geq \sup _{\pi \in P(r, t)} L(\pi, f, g)
$$

When

$$
\inf _{\pi \in P(r, t)} U(\pi, f, g)=\sup _{\pi \in P(r, t)} L(\pi, f, g)
$$

we denote their common value by $\int_{r}^{t} f(x) d g(x)$. This value is called the RiemannStieltjes integral of $f$ with respect to $g$ on $[r, t]$. It is known that $\int_{r}^{t} f(x) d g(x)$ exists whenever $f$ is continuous ${ }^{7}$.

For $r, t \in T$ with $r>t$, a partition from $r$ to $t$ is a path $\pi=\left(t_{0}, \ldots, t_{k}\right)$ with

$$
r=t_{0}>t_{1}>\cdots>t_{k-1}>t_{k}=t .
$$

Let $P(r, t)$ be the collection of partitions from $r$ to $t$. We define $M_{i}, m_{i}$ and $\Delta g_{i}$ as before, but now

$$
L(\pi, f, g)=\sum_{i=0}^{k-1} M_{i} \cdot \Delta g_{i} \quad \text { and } \quad U(\pi, f, g)=\sum_{i=0}^{k-1} m_{i} \cdot \Delta g_{i}
$$

Again, it is straightforward to check that $\inf _{\pi \in P(r, t)} U(\pi, f, g)$ and $\sup _{\pi \in P(r, t)} L(\pi, f, g)$ exist, and that

$$
\inf _{\pi \in P(r, t)} U(\pi, f, g) \geq \sup _{\pi \in P(r, t)} L(\pi, f, g)
$$

When

$$
\inf _{\pi \in P(r, t)} U(\pi, f, g)=\sup _{\pi \in P(r, t)} L(\pi, f, g)
$$

\footnotetext{
${ }^{7}$ See Rudin [21], p.125.
} 
we denote their common value by $\int_{t}^{r} f(x) d g(x)$. It is straightforward to check that

$$
\int_{r}^{t} f(x) d g(x)=-\int_{t}^{r} f(x) d g(x) .
$$

In principle, this is equivalent to a definition of the Riemann-Stieltjes integral when $g$ is non-increasing.

Theorem A.1 Let $\phi:[A, B] \rightarrow[a, b]$ be strictly increasing and continuous. Let $\alpha$ be an increasing function on $[a, b]$. Let $f$ be Riemann-Stieltjes integrable on $a, b]$ w.r.t. $\alpha$. For $y \in[A, B], \beta(y)=\alpha(\phi(y))$ and $g(y)=f(\phi(y))$. Then $g$ is Riemann-Stieltjes integrable w.r.t. $g$, and

$$
\int_{A}^{B} g(y) d \beta(y)=\int_{a}^{b} f(x) d \alpha(x) .
$$

\section{B Appendix. Proof of Theorem 6.3}

This section is devoted to the proof of Theorem 6.3. Let $\sigma$ be a continuous and strictly increasing strategy for the sender. Then the function $h(\sigma): T \rightarrow \mathbb{R}$ defined by $h(\sigma)(t)=c_{s}(\sigma(t), t)$ is continuous in $t$ by assumption [7]. Hence, the Riemann-Stieltjes integral $\int_{r}^{t} h(\sigma)(x) d \sigma(x)$ exists for all $r, t \in T$ with $r \neq t$.

Lemma B.1 Suppose that $\sigma$ is strictly increasing and $c$ has decreasing differences. Then for any $r, t \in T$ with $r \neq t$,

$$
\operatorname{dist}(\sigma)(r, t)=\inf _{\pi \in P(r, t)} \text { length }(l(\sigma))(\pi)
$$

Proof. We prove the statement in case $r<t$. The proof for the case where $r>t$ runs along similar lines. Since by definition

$$
\operatorname{dist}(\sigma)(r, t)=\inf _{\pi \in \Pi(r, t)} \operatorname{length}(l(\sigma))(\pi)
$$

it is obvious that

$$
\operatorname{dist}(\sigma)(r, t) \leq \operatorname{length}(l(\sigma))(\pi)
$$

for any partition $\pi$ from $r$ to $t$. To show the reverse inequality, by definition of $\operatorname{dist}(\sigma)(r, t)$ there exists a path $\pi=\left(t_{0}, \ldots, t_{n}\right)$ in $H$ from $r$ to $t$ such that

$$
\operatorname{length}(l(\sigma))(\pi)<\operatorname{dist}(\sigma)(r, t)+\varepsilon \text {. }
$$


Now suppose there is a $k$ with $t_{k}<t_{k+1}$ and $t_{k+1}>t_{k+2}$. It follows from monotonicity and decomposition monotonicity that $\operatorname{length}(\rho) \leq \operatorname{length}(\pi)$ for the path

$$
\rho=\left(t_{0}, \ldots, t_{k}, t_{k+2}, \ldots, t_{n}\right)
$$

Iteration of this argument yields a partition $\pi^{*}$ from $r$ to $t$ with length $(l(\sigma))\left(\pi^{*}\right) \leq$ length $(l(\sigma))(\pi)$.

Lemma B.2 Suppose that $\sigma$ is continuous and strictly increasing. Then, for any partition $\pi$ from $r$ to $t$,

$$
L(\pi, h, \sigma) \leq \operatorname{length}(l(\sigma))(\pi) \leq U(\pi, h, \sigma) .
$$

Proof. We construct the proof for the case where $r<t$. Again, the remaining case where $r>t$ runs along similar lines. Take a partition $\pi=\left(t_{0}, \ldots, t_{k}\right)$ from $r$ to $t$. Take $i$ fixed. By assumption [6], the map $y \mapsto c_{s}\left(y, t_{i}\right)$ is continuous on the interval $\left[\sigma\left(t_{i}\right), \sigma\left(t_{i+1}\right)\right]$. Write $m_{i}=\min \left\{c_{s}\left(y, t_{i}\right) \mid \sigma\left(t_{i}\right) \leq y \leq \sigma\left(t_{i+1}\right)\right\}$. Now, since $\sigma$ is strictly increasing,

$$
\begin{aligned}
\operatorname{length}(l(\sigma))(\pi) & =\sum_{i=0}^{k-1} l(\sigma)\left(t_{i}, t_{i+1}\right) \\
& =\sum_{i=0}^{k-1}\left[c\left(\sigma\left(t_{i+1}\right), t_{i}\right)-c\left(\sigma\left(t_{i}\right), t_{i}\right)\right] \\
& \geq \sum_{i=0}^{k-1} m_{i} \cdot\left[\sigma\left(t_{i+1}\right)-\sigma\left(t_{i}\right)\right]
\end{aligned}
$$

On the other hand,

$$
L(\pi, h, \sigma)=\sum_{i=0}^{k-1} n_{i} \cdot\left[\sigma\left(t_{i+1}\right)-\sigma\left(t_{i}\right)\right]
$$

where $n_{i}=\min \left\{c_{s}\left(\sigma(x), t_{i}\right) \mid t_{i} \leq x \leq t_{i+1}\right\}$. Since $\sigma$ is continuous and strictly increasing, it follows that $m_{i}=n_{i}$ for all $i$. Hence, length $(l(\sigma))(\pi) \geq U(\pi, h, \sigma)$. Similarly, length $(l(\sigma))(\pi) \leq U(\pi, h, \sigma)$.

For two partitions $\pi=\left(t_{1}, \ldots, t_{k}\right)$ and $\rho=\left(r_{1}, \ldots, r_{m}\right)$, we write $\pi \vee \rho$ for the (unique) partition $\left(u_{1}, \ldots, u_{p}\right)$ with

$$
\left\{u_{1}, \ldots, u_{p}\right\}=\left\{r_{1}, \ldots, r_{m}\right\} \cup\left\{t_{1}, \ldots, t_{k}\right\}
$$


Lemma B.3 Let $\sigma$ be strictly increasing and continuous. Suppose that c has decreasing differences. Then

$$
\sup _{\pi \in P(r, t)} L(\pi, h, \sigma) \leq \operatorname{dist}(\sigma)(r, t) .
$$

Proof. By Lemma 4.3, $l(\sigma)$ is decomposition monotone. So, length $(l(\sigma))(\pi \vee$ $\rho) \leq \operatorname{length}(l(\sigma))(\pi)$ for any $\pi, \rho \in P(r, t)$. Since also $L(\pi, h, \sigma) \leq L(\pi \vee \rho, h, \sigma)$ for any $\pi, \rho \in P(r, t)$, we get the result by Lemma B.2.

Lemma B.4 Let $\sigma$ be strictly increasing and continuous. Suppose that $c$ has decreasing differences. Then for all $r, t \in T$ with $r \neq t$,

$$
\int_{r}^{t} h(\sigma)(x) d \sigma(x)=\operatorname{dist}(\sigma)(r, t)
$$

Proof. By Lemmas B.1 and B.2,

$$
\int_{r}^{t} h(\sigma)(x) d \sigma(x)=\inf _{\pi \in P(r, t)} U(\pi, h, \sigma) \geq \inf _{\pi \in P(r, t)} \operatorname{length}(l(\sigma))(\pi)=\operatorname{dist}(\sigma)(r, t) .
$$

On the other hand, by Lemma B.3,

$$
\int_{r}^{t} h(\sigma)(x) d \sigma(x)=\sup _{\pi \in P(r, t)} L(\pi, h, \sigma) \leq \operatorname{dist}(\sigma)(r, t) .
$$

This completes the proof.

Lemma B.5 Let $\sigma$ be an SE strategy. Suppose that c has decreasing differences.

Then for all $t \in T$,

$$
\alpha(t)=\int_{\underline{t}}^{t} h(\sigma)(x) d \sigma(x) .
$$

Proof. Take $r, t \in T$ with $r \neq t$. We show that

$$
\alpha(t)-\alpha(r)=\int_{r}^{t} h(\sigma)(x) d \sigma(x)
$$

The claim then follows by taking $r=\underline{t}$ and using the fact that $\alpha(\underline{t})=0$. Take $r, t$ with $r \neq t$. Let $\pi=\left(t_{0}, \ldots, t_{k}\right)$ be a partition from $r$ to $t$. Then

$$
\begin{aligned}
\alpha(t)-\alpha(r) & =\sum_{i=0}^{k-1}\left(\alpha\left(t_{i+1}\right)-\alpha\left(t_{i}\right)\right) \\
& \leq \sum_{i=0}^{k-1} l(\sigma)\left(t_{i}, t_{i+1}\right) \\
& =\operatorname{length}(l(\sigma))(\pi)
\end{aligned}
$$


Hence, by Lemma B.1 and Lemma B.4

$$
\alpha(t)-\alpha(r) \leq \inf _{\pi \in P(r, t)} \operatorname{length}(l(\sigma))(\pi)=\int_{r}^{t} h(\sigma)(x) d \sigma(x) .
$$

This also implies that

$$
\alpha(r)-\alpha(t) \leq \int_{t}^{r} h(\sigma)(x) d \sigma(x)=-\int_{r}^{t} h(\sigma)(x) d \sigma(x)
$$

so that

$$
\int_{r}^{t} h(\sigma)(x) d \sigma(x) \leq \alpha(t)-\alpha(r)
$$

This completes the proof.

\section{Appendix. Proof of Theorem 6.4}

This section is devoted to the proof of Theorem 6.4. We prove the following fact. The proof is loosely based on a similar proof by Peano.

Theorem C.1 Let $V=\mathbb{R}_{+} \times \mathbb{R}_{+}$. Suppose $H: V \rightarrow \mathbb{R}$ is continuous and bounded. Then there exists at least one solution of the initial value problem

$$
f^{\prime}(s)=H(s, f(s)) \text { with } f(0)=0 .
$$

Moreover, any solution is automatically continuously differentiable.

Proof. For $n, k \in \mathbb{N}$ define $s_{n, k}=k \cdot 2^{-n}$. Now take $n \in \mathbb{N}$ fixed for the moment. We set $x_{n, 0}=0$, and for $k \in \mathbb{N}$

$$
x_{n, k+1}=x_{n, k}+2^{-n} \cdot H\left(s_{n, k}, x_{n, k}\right) .
$$

Define $f_{n}: \mathbb{R}_{+} \rightarrow \mathbb{R}$ by, for $s \in\left[s_{n, k}, s_{n, k+1}\right)$,

$$
f_{n}(s)=x_{n, k}+2^{n} \cdot\left(s-s_{k}\right) \cdot\left(x_{n, k+1}-x_{n, k}\right) .
$$

Let $M>0$ be such that $|H(s, a)| \leq M$ for all $(s, a) \in V$. First we show for all $s, s^{\prime} \in \mathbb{R}_{+}$that

$$
\left|f_{n}\left(s^{\prime}\right)-f_{n}(s)\right| \leq M \cdot\left|s^{\prime}-s\right| .
$$

Take $k, m$ such that $s \in\left[s_{n, k}, s_{n, k+1}\right)$ and $s^{\prime} \in\left[s_{n, m}, s_{n, m+1}\right)$. Then $f_{n}(s)$ is a linear interpolation between $x_{n, k}$ and $x_{n, k+1}$, and $f_{n}\left(s^{\prime}\right)$ is a linear interpolation between $x_{n, m}$ and $x_{n, m+1}$. Therefore it suffices to prove that

$$
\left|x_{n, k+i}-x_{n, k}\right| \leq M \cdot\left|s_{n, k+i}-s_{n, k}\right| .
$$


This however immediately follows from the observation that

$$
\begin{aligned}
\left|x_{n, k+1}-x_{n, k}\right| & =\left|2^{-n} \cdot H\left(s_{n, k}, x_{n, k}\right)\right| \\
& \leq 2^{-n} \cdot M \\
& =M \cdot\left|s_{n, k+1}-s_{n, k}\right| .
\end{aligned}
$$

Now write

$$
D=\left\{k \cdot 2^{-n} \mid k, n \in \mathbb{N}\right\} .
$$

Obviously $D$ is a countable set. Let $d_{1}, d_{2}, \ldots$ be an enumeration of $D$. Consider $d_{1} \in D$. Then for all $n$ we have $\left|f_{n}\left(d_{1}\right)\right| \leq M \cdot d_{1}$. So, there is a subsequence $\left(f_{n}^{1}\right)_{n=1}^{\infty}$ of $\left(f_{n}\right)_{n=1}^{\infty}$ for which $\left(f_{n}^{1}\left(d_{1}\right)\right)_{n=1}^{\infty}$ is convergent. Iteratively for $k=$ $1,2, \ldots$ we can take a subsequence $\left(f_{n}^{k}\right)_{n=1}^{\infty}$ of $\left(f_{n}^{k-1}\right)_{n=1}^{\infty}$ for which $\left(f_{n}^{k}\left(d_{l}\right)\right)_{n=1}^{\infty}$ is convergent for all $l=1, \ldots k$. Then for the sequence $\left(f_{k}^{k}\right)_{k=1}^{\infty}$ we have that $\left(f_{k}^{k}(d)\right)_{k=1}^{\infty}$ is convergent for all $d \in D$.

Write $g_{k}=f_{k}^{k}$. Take any $s \geq 0$. We argue that $\left(g_{k}(s)\right)_{k=1}^{\infty}$ is Cauchy. Take $\varepsilon>0$. Since $D$ is dense in $\mathbb{R}_{+}$, we can take a $d \in D$ with $|s-d|<\frac{\varepsilon}{3 M}$. Further, since $\left(g_{k}(d)\right)_{k=1}^{\infty}$ is convergent, we can take $K>0$ such that

$$
\left|g_{k}(d)-g_{m}(d)\right|<\frac{\varepsilon}{3}
$$

whenever $k, m>K$. Then, for $k, m>K$,

$$
\begin{aligned}
\left|g_{k}(s)-g_{m}(s)\right| & =\left|g_{k}(s)-g_{k}(d)+g_{k}(d)-g_{m}(d)+g_{m}(d)-g_{m}(s)\right| \\
& \leq\left|g_{k}(s)-g_{k}(d)\right|+\left|g_{k}(d)-g_{m}(d)\right|+\left|g_{m}(d)-g_{m}(s)\right| \\
& <M \cdot|s-d|+\frac{\varepsilon}{3}+M \cdot|s-d| \\
& \leq M \cdot \frac{\varepsilon}{3 M}+\frac{\varepsilon}{3}+M \cdot \frac{\varepsilon}{3 M}=\varepsilon .
\end{aligned}
$$

Define $f(s)=\lim _{n \rightarrow \infty} g_{n}(s)$. We claim that $f$ is continuous. Take any $s, s^{\prime} \geq 0$. Since $\left(g_{n}\right)_{n=1}^{\infty}$ is a subsequence of $\left(f_{n}\right)_{n=1}^{\infty}$, we know from the argument above that

$$
\left|g_{n}\left(s^{\prime}\right)-g_{n}(s)\right| \leq M \cdot\left|s^{\prime}-s\right|
$$

for all $n$. The claim now follows by taking limits for $n \rightarrow \infty$.

So, the function $s \mapsto H(s, f(s))$ is continuous. Then it is integrable on bounded intervals. Take any $s \geq 0$. We show that

$$
f(s)=\int_{0}^{s} H(u, f(u)) d u .
$$


Take $n \in \mathbb{N}$ fixed. Let $p(n)$ be the natural number such that $g_{n}=f_{n}^{n}=f_{p(n)}$.

Define

$$
h_{n}(s)=H\left(s_{p(n), k}, x_{p(n), k}\right) \quad \text { if } s \in\left[s_{p(n), k}, s_{p(n), k+1}\right)
$$

Then clearly $g_{n}(s)=\int_{0}^{s} h_{n}(u) d y$. We argue that $h_{n}(s) \rightarrow H(s, f(s))$ as $n \rightarrow \infty$.

First note that

$h_{n}\left(s_{p(n), k}\right)=H\left(s_{p(n), k}, x_{p(n), k}\right)=H\left(s_{p(n), k}, f_{p(n)}\left(s_{p(n), k}\right)\right)=H\left(s_{p(n), k}, g_{n}\left(s_{p(n), k}\right)\right)$.

Take $s \geq 0$ arbitrary. Choose $k(n)$ such that $s \in\left[s_{p(n), k(n)}, s_{p(n), k(n)+1}\right)$. Then

$$
h_{n}(s)=H\left(s_{p(n), k(n)}, x_{p(n), k(n)}\right)=H\left(s_{p(n), k(n)}, g_{n}\left(s_{p(n), k(n)}\right)\right)
$$

Since clearly $p(n) \rightarrow \infty$ as $n \rightarrow \infty$, we know that $s_{p(n), k(n)} \rightarrow s$ as $n \rightarrow \infty$.

Therefore it suffices to show that

$$
g_{n}\left(s_{p(n), k(n)}\right) \rightarrow f(s) .
$$

This however follows from the observations that $g_{n}(s) \rightarrow f(s)$ and $\mid g_{n}\left(s^{\prime}\right)-$ $g_{n}(s)|\leq M \cdot| s^{\prime}-s \mid$.

Hence, since $\left|h_{n}(s)\right| \leq M$ for all $s$ and $n$, the theorem of bounded convergence yields

$$
f(s)=\lim _{n \rightarrow \infty} g_{n}(s)=\lim _{n \rightarrow \infty} \int_{0}^{s} h_{n}(u) d u=\int_{0}^{s} H(u, f(u)) d u .
$$

This completes the proof.

Now we are ready to show Theorem C.2. A subset $I$ of $S$ is called an initial subset if for all $s \in I$ and $s^{\prime} \in S$ with $s^{\prime}<s$ we have $s^{\prime} \in I$. A function $g: I \rightarrow \mathbb{R}$ is called a partial solution on $I$ if $g(0)=0, g$ is differentiable on $I$, and

$$
g^{\prime}(s)=H(s, g(s))
$$

for all $s \in I$.

Theorem C.2 Suppose that $H: S \times A \rightarrow \mathbb{R}$ is continuous, bounded, and nonincreasing in the second coordinate. Then the initial value problem

$$
f^{\prime}(s)=H(s, f(s)) \text { with } f(0)=0
$$

has a unique solution $f^{*}$. Moreover, for any partial solution $g$ on $I$ it holds that $f^{*}(s)=g(s)$ for all $s \in I$. 
Proof. By Theorem C.1, there exists a solution to the initial value problem. Suppose that both $f$ is a solution of the initial value problem, and that $g$ is a partial solution on $I$. It suffices to show that $f^{*}(s)=g(s)$ for all $s \in I$.

For $s \in I$, define $h(s)=f(s)-g(s)$. We show that $h(s)=0$ for all $s \in I$. Suppose that $h(s)>0$ for some $s \in I$. Since $h(0)=0$, obviously $s>0$. Define

$$
s^{*}=\inf \{x \mid 0 \leq x \leq s \text { and } h(y)>0 \text { for all } y \in(x, s)\} .
$$

Since $h$ is continuous, $h(s)>0$, and $h(0)=0$, we know that $s^{*}<s$ and $h\left(s^{*}\right)=0$. So, since $h$ is continuously differentiable, by the mean value theorem there exists $\tau \in\left(s^{*}, s\right)$ with

$$
h^{\prime}(\tau)=\frac{h(s)-h\left(s^{*}\right)}{s-s^{*}}=\frac{h(s)}{s-s^{*}}>0 .
$$

However, $\tau \in\left(s^{*}, s\right)$ implies that $h(\tau)>0$ by definition of $s^{*}$. Then $f(\tau)>g(\tau)$. So, since $H$ is non-increasing in the second argument, $f^{\prime}(\tau)=H(\tau, f(\tau)) \leq$ $H(\tau, g(\tau))=g^{\prime}(\tau)$. Hence, $h^{\prime}(\tau) \leq 0$. Contradiction. 